\title{
GLAD!
}

Revue sur le langage, le genre, les sexualités

$05 \mid 2018$

Raconter les sexualités depuis la marge

\section{Service domestique, sexualité, race et héritages coloniaux à l'île Maurice}

Une démarche exploratoire

Domestic Service, Sexuality, Race, and Colonial Heritage in Mauritius - An

Exploratory Approach

\section{Colette Le Petitcorps}

\section{OpenEdition}

Journals

Édition électronique

URL : http://journals.openedition.org/glad/1212

ISSN : 2551-0819

Éditeur

Association GSL

Référence électronique

Colette Le Petitcorps, "Service domestique, sexualité, race et héritages coloniaux à l'île Maurice », GLAD! [En ligne], 05 | 2018, mis en ligne le 15 décembre 2018, consulté le 17 décembre 2020. URL: http://journals.openedition.org/glad/1212

Ce document a été généré automatiquement le 17 décembre 2020.

\section{cc) (1) $\odot$}

La revue GLAD! est mise à disposition selon les termes de la Licence Creative Commons Attribution Pas d'Utilisation Commerciale - Pas de Modification 4.0 International. 


\title{
Service domestique, sexualité, race et héritages coloniaux à l'île Maurice
}

\author{
Une démarche exploratoire \\ Domestic Service, Sexuality, Race, and Colonial Heritage in Mauritius - An \\ Exploratory Approach
}

Colette Le Petitcorps

1 Le travail sexuel constitue une éventualité du service domestique ${ }^{1}$. Il fait partie des possibles du travail de la bonne "à tout faire " et de son avatar contemporain, la femme de ménage. Comme le souligne Christine Delphy, le travail à domicile, effectué à grande majorité par les femmes, ne doit pas être défini à partir des tâches qui le composent, mais plutôt comme une certaine relation de travail, un rapport de production (Delphy, 1978: 53). Si Delphy limite la définition du travail ménager au travail non payé dans le cadre familial, Colette Guillaumin inclut dans l'analyse de l'exploitation des femmes l'ensemble des modes concrets d'appropriation des femmes par les hommes (Guillaumin 1992). Ceux-ci incluent outre l'exploitation de leur force de travail, l'appropriation de leur corps, unité matérielle productrice de la force de travail. L'obligation sexuelle est l'une des expériences concrètes du sexage (Guillaumin 1992). Elle se retrouve, comme l'explicite Paola Tabet avec le concept d'" échange économicosexuel », dans toutes les activités féminines (Tabet 2004). L'échange économico-sexuel, qui désigne l'échange d'un ou plusieurs actes sexuels contre une compensation (argent, logement, statut social, etc.), lie les prostituées et les femmes mariées dans un même continuum de la domination. Il est une manifestation du rapport social de sexe et des disparités économiques. Les relations des femmes à la sexualité comme source de revenus dépendent des conditions matérielles auxquelles elles sont soumises (Moujoud 2007) par les mécanismes du capitalisme global. Les abus sexuels divers des hommes sur les femmes employées à domicile (de l'exhibition de la nudité ou d'une masturbation, au harcèlement sexuel et au viol) sont des pratiques qui s'appuient sur des représentations de la disponibilité sexuelle des femmes travaillant à domicile qui 
héritent de la longue histoire du viol des femmes comme arme de domination, y compris coloniale (Martin-Fugier 1979 ; Corossacz 2014 ; Davis 1983 ; hooks 2015).

2 Enquêter sur les rapports sexuels dans les services domestiques est difficile. La honte, le socialement indicible, le regain de violence à parler d'une violence, la défiance vis-àvis de l'enquêtrice... un ensemble de conditions sociales entravent le projet de recueil d'expériences de cet aspect du travail de service domestique. Il est également possible que les rapports sexuels entre bonnes et patrons ne soient plus qu'une «légende", comme l'affirme Maria Arondo (1975) et qu'ils soient peu signifiants des rapports de service qui se nouent dans les nouvelles formes des emplois à domicile, payés à l'heure et sans logement à demeure des employeurs. Face aux difficultés de terrain, l'analyse que je propose ne cherche pas à recueillir des témoignages pour démontrer la permanence de la sexualité dans les relations et les pratiques de service domestique. Elle vise plutôt à questionner, à partir de la façon dont la sexualité apparait dans les discours et les imaginaires sur les services domestiques, la manière dont le fait de langage (Bornand \& Leguy 2013) sur l'aspect sexuel des relations de service domestique continue d'ordonner le social et de légitimer des hiérarchies. Je m'inspire pour cela de la littérature sur les imaginaires sociaux et leur rôle dans le maintien des hiérarchies sociales, ainsi que de la littérature sur le pouvoir qui réside et circule dans la prise de parole, les silences et les rumeurs (N'Gaïde 2003, White 2000, Rubenstein 2007, Bornand et Leguy 2013 notamment).

3 Le contexte de l'étude est l'̂̂le Maurice, une société qui s'est historiquement formée à partir de trois périodes coloniales (hollandaise de 1638 à 1710, puis française de 1715 à 1810 et enfin britannique de 1810 à 1968, date de l'indépendance en 1968) et autour de l'économie de plantation sucrière. Les migrations de la main-d'œuvre, esclave (à partir de la colonisation hollandaise jusque dans les années 1830) puis engagée (des années 1820 aux années 1910), ainsi que les rapports de production dans l'espace confiné des plantations, ont été organisés pour l'exportation du sucre sur le marché européen (Claveyrolas 2017). Les procédés d'assimilation et de différenciation sociale du pouvoir colonial ont d'abord mobilisé des référents raciaux sous la colonisation française (distinguant les propriétaires de concessions blancs, des esclaves noirs et des libres de couleur), puis des catégories ethniques sous la colonisation anglaise. L'administration coloniale britannique a en effet créé, pour les objectifs de la production de la plantation, des catégories de classement des travailleurs distinguant les anciens esclaves, assimilés au monde blanc (qui deviennent les "Créoles») et les Indiens engagés (qui deviennent "Indo-Mauriciens», puis « Hindous») (Chazan-Gillig 2001). Les héritages de la construction hiérarchique des différences dans la société mauricienne, tels qu'ils apparaissent dans "le jeu hasardeux des dominations" (Foucault 1971) sur la scène domestique aujourd'hui à l'île Maurice, font l'objet de mon analyse qui prend la sexualité dans le langage pour point de départ.

4 Les travaux d'Ann Laura Stoler ont souligné à quel point les aspects affectifs et charnels des relations dans l'espace domestique et intime étaient un enjeu de connaissance de l'appareil de gouvernance colonial (Stoler 2002). La surveillance de l'intimité, ainsi que la prohibition du mariage et du concubinage entre colons et colonisées sont apparues lorsque les relations sociales et sexuelles troublaient l'ordre colonial. Le contrôle de la sexualité, dans les relations de proximité inévitables entre colons et colonisées que représentent les services domestiques, a été central dans la production des catégories raciales visant à légitimer l'ordre colonial (Stoler 2002). À partir de la sexualité dans les 
services domestiques, telle qu'elle apparait dans les pratiques langagières contemporaines, il s'agit d'interroger la (ré) actualisation de catégories raciales dans les imaginaires sociaux de la hiérarchie à l'île Maurice, un processus que la notion de la « colonialité du pouvoir » permet d'examiner (Quijano 2007).

5 Les données empiriques présentées pour la démonstration rassemblent des fragments d'entretien réalisés avec un petit nombre de femmes (moins d'une dizaine sur une quarantaine d'enquêtées) dont j'ai obtenu une certaine confiance pour qu'elles parlent de la sexualité dans les relations de service domestique entre « bonnes » et " patrons ${ }^{2}$ ». Étant donné le nombre d'entretiens restreint sur lequel repose l'analyse, on s'autorisera à présenter l'analyse développée dans cet article en des termes exploratoires. Au cours d'une enquête de terrain étalée sur la longue durée (de 2013 à 2018, totalisant 7 mois), j'ai rencontré plusieurs fois des femmes, à la fois des patronnes et des bonnes, avec lesquelles j'ai noué une relation de familiarité dans leurs espaces de vie. Mon origine française, «blanche » et universitaire a facilité le dialogue avec des femmes patronnes appartenant à la communauté des descendants européens de l'île (appelés dans le langage courant «Franco-Mauriciens» ou «Blancs»). Les femmes travaillant comme bonnes qui se sont arrogé la liberté d'évoquer en entretien les dimensions sexuelles des relations de service dans leur expérience du travail ont deux caractéristiques: elles ont un discours globalement contestataire des rapports de domination vécus dans les services domestiques ${ }^{3}$, quel que soit leur âge, et elles connaissent des éléments de ma vie qui relativisent ma position de française, blanche, dominante et potentielle patronne à leurs yeux. Cela est notamment dû au fait que mon conjoint, présent durant certains de mes séjours à l'île Maurice, est issu d'une famille "créole » mauricienne. Ces femmes ont été rencontrées par des intermédiaires (des membres de la famille à laquelle je suis personnellement liée) ou de manière fortuite dans des quartiers d'habitat précaire que j'ai fréquentés quotidiennement durant mes séjours ${ }^{4}$. Elles ont donc pour point commun leur condition de classe, indépendamment de la catégorie ethnique par laquelle elles sont classées dans les représentations sociales (" créole », « hindoue » ou « musulmane »).

6 Une première approche de la façon dont les patrons, et le caractère sexuel implicite de leurs actes, sont évoqués dans les récits du travail de bonnes est abordée dans la première partie. La deuxième partie analyse le sexuel dans les services domestiques et les histoires de famille du point de vue des patronnes. La troisième partie traite des rumeurs sur les bonnes et leur sexualité, qui réactualisent la dimension raciale de l'ordre social dans la société mauricienne contemporaine.

\section{Implicite sexuel et « Monsieur blanc » dans les récits de travail de bonnes}

7 Quels que soient les générations et les parcours des femmes rencontrées, qu'elles aient 20 ou 70 ans, qu'elles aient travaillé comme bonne toute leur vie ou qu'elles aient été employées des usines avant d'entrer dans les emplois domestiques, aucune ne parle de rapports sexuels qu'elles auraient pu avoir avec leurs patrons. Elles n'évoquent pas non plus l'existence de cette pratique concernant d'autres bonnes en général. En revanche, et ce n'est qu'en réécoutant les enregistrements d'entretiens menés en kreol que je le constate, certaines femmes font brièvement allusion à des actes de leurs patrons qui simulent un rapport sexuel et leur font comprendre qu'ils « exigent » quelque chose en 
plus de leur service. De manière très discrète et elliptique, elles mentionnent des hommes qui leur touchent les fesses ou se montrent nus devant elles. Ces comportements sont présentés comme assez banals dans leur expérience du travail et transversaux à la catégorie ethnique d'appartenance sociale de ces hommes («FrancoMauricien ", «Hindou ", «Créole », " Musulman », "Sino-Mauricien» d'après les catégories reconnues par la Constitution de 1968, qui sont encore activées dans les identifications réciproques des appartenances sociales). Un des exemples revenu plusieurs fois dans les récits d'expérience du travail de bonne est celui de la douche. Le patron veut prendre sa douche, il a donc besoin d'une serviette, c'est la bonne qui doit lui apporter sa serviette, et tout se passe pour qu'elle ne puisse pas l'apporter avant qu'il ne se déshabille. Il lui est en prime reproché de ne pas avoir apporté la serviette au moment opportun. Ketty, la trentaine, travaillant dans plusieurs maisons en bord de mer, mentionne rapidement et sans détail un de ces épisodes avec le mari d'une de ses patronnes :

\footnotetext{
Madamla so monsieur. Li ti kontan exigé dimounn, surtou kan li dan lakaz, bizin okip li, prepar so zafer, so douche, so mangé, mé mwa mo condition c'est pas comme ça. Après c'est nettoyer, repasser. Et puis après mo bizin pran so zafer pou li al pran so douche, met so zafer lor la baignoire, et puis, pou evit, problem, preferable changé.

Le mari de Madame, il aimait bien exiger de la personne, surtout quand il est dans la maison, il faut s'occuper de lui, préparer ses affaires, sa douche, son repas, mais moi mes conditions c'est pas comme ça. Moi je nettoie je repasse. Et puis après je dois prendre ses affaires pour qu'il prenne sa douche, je dois mettre ses affaires sur la baignoire, et puis, pour éviter les problèmes, j'ai trouvé préférable de changer [de travail].
}

8 Les femmes comprennent ces actes comme des tests qui, si elles jouent le jeu et acceptent d'apporter la serviette à chaque fois, conduisent à étendre la nature des services exigés d'elles et de leur corps. Ils leur font également sentir que leur statut de bonne de maison les rabaisse à se frotter à la proximité du corps, nu, suant, au réveil, des hommes qui, avec leur épouse, achètent leurs services. Ces scènes rejouent les rapports d'appropriation de service domestique dont l'histoire longue remonte à l'esclavage, à l'engagisme et à la colonisation à l'île Maurice. Les femmes qui mentionnent cette expérience du travail disent toutes avoir quitté la maison où cela s'est produit. Elles restent tout aussi elliptiques sur la façon dont elles s'en sont expliquées à la patronne.

9 Ces quelques mots lâchés au fil de nos discussions sont à considérer en tenant compte, plus largement, du discours que les femmes qui travaillent comme bonnes tiennent à l'égard des patrons, ou plus précisément du mari de "Madame». "Madamla so monsieur», "mo pé travay dan lakaz Madam ${ }^{5}$ ", ces expressions du kreol sont éloquentes de la place de l'homme dans la triade de la relation de service domestique : il n'a pas la responsabilité d'être le patron dans la maison. Mais il est là. Et bien qu'une ample littérature sur les services domestiques n'ait insisté que sur les relations de travail entre femmes, employeuses et travailleuses domestiques (Rollins 1990, Romero 1992, Fraisse 1979, Brites 2000, Fox-Genovese 1988, Cock 1989, pour n'en citer que quelques-unes), les femmes que j'ai rencontrées à l'île Maurice donnent un rôle important aux hommes dans leur expérience du travail de service. Or les portraits qu'elles en font sont souvent ambigus. Elles décrivent à la fois de "vrais cochons", mais il existe aussi des «Monsieur " "très gentils ». La gentillesse est un attribut 
systématiquement réservé au "Monsieur ", plutôt qu'à la «Madame » qui donne les consignes domestiques (ou les ordres). Si celle-ci n'est pas maltraitante ou acariâtre, elle est tout au plus "korek» (correcte). Ce qui se comprend au fil des discours recueillis est que la gentillesse est concrètement attribuée au «Monsieur » qui donne de l'argent en plus de la rémunération du travail fixée par la patronne, ce dont la patronne n'a souvent pas connaissance. Il n'est jamais dit si et contre quels types de service cet argent est échangé. Les hommes qui sont plus certainement réputés pour être les plus " gentils " parmi les femmes rencontrées actuellement sont les «Blancs » et les étrangers français qui sont installés à l'île Maurice.

La présence des hommes dans les expériences de travail relatées par des femmes employées comme bonnes amène à considérer l'existence d'un imaginaire de la mobilité sociale que l'argent, supplémentaire à la paie, du "Monsieur ", "Blanc", rendrait possible. Cet imaginaire est plutôt partagé par des femmes de l'enquête qui ont déjà plus de 50 ans et qui ont connu les formes les plus traditionnelles du service domestique : à temps plein, auprès d'une même famille, pendant de longues années, en logeant parfois dans la " dépendance ${ }^{6}$ ». L'imaginaire des possibles que le "Monsieur blanc " provoque est par exemple présent dans les propos d'Odile, 65 ans, travailleuse domestique depuis ses 17 ans, qui a logé dans les dépendances jusque dans les années 2000. Elle me raconte une anecdote avec un «Monsieur» il y a 20 ans de cela. Dans la première version de l'anecdote - nous venons alors de nous rencontrer et elle me raconte sa vie dans les grands traits telle qu'elle l'appréhende rétrospectivement -, le Monsieur est déprimé par sa femme qui le trompait : il rentre alors un jour dans la cuisine pour frotter son pénis contre ses fesses. L'allusion est rapide et Odile me présente ce type de patron comme un "cochon». Je noue avec Odile une relation d'amitié au fil des jours et elle revient après quelques semaines sur cette anecdote, durant une entrevue informelle où nous mangeons dans un snack. Cette fois-ci la présentation de l'anecdote est différente. Elle raconte qu'elle a fait comme si de rien n'était parce qu'elle ne savait pas qu'elle pouvait intéresser un homme blanc. Elle n'avait à l'époque pas d'estime d'elle-même, il ne s'est donc finalement rien passé. Il s'agit dans son récit non pas d'un moment désagréable, mais plutôt d'une occasion manquée. C'est un sentiment qu'elle avoue avec l'évolution de notre relation, mais qui reste difficile à exprimer par crainte du jugement social. On devine aussi qu'il pouvait y avoir un intérêt à accepter les avances de son patron pour améliorer quelque chose dans sa vie.

11 Pour comprendre le raisonnement d'Odile et ce qu'elle aurait gagné à avoir une relation avec le patron, il est nécessaire d'examiner les expériences d'autres femmes interviewées. Plusieurs femmes ont effectivement reçu de l'argent de certains de leurs patrons, ce qui leur a permis d'obtenir une ressource supplémentaire pour assurer la survie des membres de leur foyer et envisager une scolarité pour leurs enfants. L'argent obtenu du patron a parfois aidé à construire leur maison, à acheter une "maison du gouvernement $»^{7}$ ou/et à payer les dépenses scolaires de leurs enfants (cours particuliers, écoles professionnelles ou études supérieures). Les niveaux de rémunération des travailleuses domestiques fixés par le gouvernement depuis une loi de $1983^{8}$ et par les patronnes à l'embauche restent insuffisants pour couvrir l'ensemble des dépenses des foyers. Or les femmes contribuent de plus en plus à payer ces dépenses avec l'augmentation des niveaux de consommation et la croissance du chômage des hommes. C'est dans ce contexte que l'argent donné par le patron en sousmain constitue une forme de revenu du travail qui compte. Ce que la bonne doit rendre 
comme service en échange correspond à l'ellipse majeure des récits d'expérience recueillis : tout ne se dit pas dans ce qui tient le système de débrouille.

Il semble cependant que les femmes les plus jeunes de l'enquête, qui ont entre 20 et 40 ans, comptent moins sur l'argent du patron que leurs aînées pour envisager les conditions de reproduction de leur famille. Celles-ci sont entrées sur le marché des emplois domestiques au moment où les dépendances étaient démolies, et où le nombre d'heures de service d'une bonne consommées par les familles se réduisait. Le fait que la bonne ne soit aujourd'hui plus entièrement intégrée à la vie de la maisonnée (Simmel 1999) constitue un changement dans l'intimité partagée dans l'espace domestique, dans la relation de service et dans les formes de l'appropriation des femmes en tant que bonnes. La part de la rémunération rétribuée par un seul couple de patrons, que ce soit la paie de Madame ou celle de Monsieur, a diminué parmi les diverses ressources que les femmes tentent de réunir pour faire survivre leur foyer. Il est donc fort possible que l'imaginaire associé à la mobilité sociale par le «Monsieur blanc» disparaisse avec la génération de travailleuses domestiques qui partent aujourd'hui à la retraite. Cet imaginaire hérite de l'histoire connue de l'ascension sociale et de l'accès à la terre de femmes qui étaient les maitresses des «maîtres blancs », ce que j'aborde dans la partie qui suit.

13 Les expériences du travail relatées par des bonnes ne font pas de mention explicite de la sexualité dans les services domestiques. Elles donnent en revanche une place importante au patron, autour duquel sont tenus des propos ambigus, s'installent des non-dits et se reproduisent certains imaginaires sociaux. Le patron qui donnerait potentiellement une rémunération supplémentaire à celle de la patronne, contre un service qui demeure opaque dans les discours, a un visage racial: il est blanc. Les espoirs (ou expériences passées) de voir les perspectives de sa famille, et en particulier de ses enfants, s'améliorer restent attachés, pour les femmes les plus âgées de l'enquête, au lien intime qui se noue dans la maison avec le «Monsieur blanc ». Celui-ci est placé au sommet de la hiérarchie sociale subjectivée. Par contre, les femmes plus jeunes de l'enquête ne semblent pas avoir les mêmes attentes envers leurs patrons, qui ont des origines ethniques plus variées et qu'elles côtoient moins dans les emplois domestiques actuels. L'ensemble des représentations de la hiérarchie sociale/raciale et des manières d'évoluer avec elle dans la société s'en trouve chamboulé.

\section{Maîtres et servantes, race et sexualité dans les discours de patronnes}

Qu'en est-il du point de vue de patronnes sur les rapports sexuels éventuels entre bonnes et époux? Lorsque je leur demande de raconter leur histoire, en décrivant particulièrement leurs relations aux domestiques tout au long de leur vie, certaines patronnes rencontrées, qui se désignent comme «Franco-Mauriciennes» ou «mulâtres », font ouvertement mention des rapports sexuels qu'il y a pu avoir entre «maîtres » et « servantes ${ }^{9}$ ». Elles évoquent ces rapports sans nécessairement que je les y invite. En revanche, ce n'est pas en tant qu'épouse d'un homme qui a couché avec la servante qu'elles parlent. Les rapports sexuels entre maître et servantes font toujours référence à un passé, dépassé, qui implique l'ensemble de la communauté francomauricienne et qui peut concerner plus particulièrement leur histoire familiale, même si les faits renvoient plutôt à leurs ancêtres. 

d'une femme issue du groupe des colonisateurs européens les plus fortunés. Mme R. est l'épouse d'un propriétaire sucrier à la retraite. Le couple vit dans deux grandes et belles maisons, l'une dans un quartier résidentiel urbain chic, l'autre en bord de mer. Leurs possessions foncières les classent dans les représentations sociales parmi les «Grands Blancs ", qui se distinguent des "Ti Blancs", lesquels n'ont pas d'autre propriété foncière que leur maison ou leur appartement. Mme R. doit avoir autour des 70 ans, a peu d'expérience professionnelle et c'est elle qui gère le personnel domestique, composé de femmes et d'hommes : un majordome, deux servantes, une cuisinière, un chauffeur, un gardien et des jardiniers. Dans une première entrevue qui a lieu dans sa maison côtière, sous la varangue faisant face à la mer, Mme R. me fait part de ses activités liées aux tentatives de conservation du patrimoine de l'histoire coloniale mauricienne que l'État veut raser depuis quelques années. Sa conception du patrimoine mauricien, dont le Franco-Mauricien planteur sucrier est le créateur, inclut le métissage qu'elle constate aujourd'hui dans la population mauricienne. Ce métissage est explicitement décrit comme étant le fruit des rapports sexuels entre les maîtres, les fils des maîtres et les servantes qui ont donné lieu à des enfants illégitimes.

Mme R. : Je m'occupe beaucoup de patrimoine, essayer de sauver ce qui peut être sauvé. C'est un désastre. De toute façon ils veulent vraiment effacer la présence française à Maurice. C'est dommage, parce qu'on est vraiment multiracial, et on a cet apport de français, de l'anglais, hindou, chinois, créole, de l'Afrique, on a des coutumes, des croyances, des superstitions, de Madagascar, très fort, chez les créoles qui font du vaudou, c'est dommage, on perd ça... Tout le monde est pareil. C'est dommage parce que le FrancoMauricien est très responsable de ce qui se passe. Il a réussi dans le sucre, et en plus on est un peuple très métissé. (...) Il y a beaucoup de familles, que je connais, une très vieille famille implantée mauricienne a épousé une Indienne ! Maintenant, ça s'est ouvert, tout le monde a épousé tout le monde, ma grand-mère n'acceptait pas qu'une personne noire entre chez elle ! Enquêtrice : C'est plus mal vu.

Mme R.: Non. C'est pas question mal vu, c'est surtout, est-ce que deux cultures pourront, c'est pas deux personnes mais deux familles qu'on épouse des deux côtés. (...) Toutes les familles, moi qui fais beaucoup de généalogie, premièrement, il y a énormément d'enfants illégitimes, beaucoup beaucoup. Beaucoup beaucoup. Donc un Musulman peut descendre d'un Chinois, un Européen peut descendre d'un Créole... c'est une très grande richesse.

$\mathrm{E}:$ Et vous avez connaissance des familles de ces enfants illégitimes?

Mme R. : Ah oui !... Je connais la généalogie, même les grandes familles, je sais. J'ai les papiers, j'ai les actes, les reconnaissances de paternité. J'ai un cousin, ça s'est passé comme ça, il a dit «non, je ne veux pas savoir ! C'est faux !» (...) Ça fait six ans je fais une recherche sur une famille illustre, c'est très certainement un enfant issu du maître et de la servante. C'était toujours le maître et les bonnes, presque toujours, rarement la maîtresse. A part deux ou trois cas où la maîtresse a eu un enfant avec un Malgache, domestique (rit), cuisinier en plus, qui lui faisait des bons plats sûrement! Mais sinon c'était l'inverse, et les fils! Les fils ! Ça c'est pas inventé ${ }^{10}$.

Les propos de Mme R. illustrent la forme d'appropriation de la mémoire du passé colonial par les Franco-Mauriciens : ils sont les fondateurs de la colonie, les acteurs principaux de l'Histoire, de l'économie (Boudet, Peghini 2008), y compris de la diversité « raciale et culturelle » de la population. Cette mémoire est différente de celle cultivée par l'État qui cherche plutôt à rompre avec l'histoire coloniale et à rattacher l'histoire 
de l'île Maurice à celle du groupe immigrant indien (Bouget \& Peghini 2008). La mémoire franco-mauricienne vise à légitimer au contraire la centralité de l'industrie sucrière dans la cohésion nationale (Ibid.). Ce que souligne Mme R. en revanche, dont la littérature sur les mémoires à l'île Maurice ne parle pas, est que le Franco-Mauricien est à l'origine de l'économie (le sucre) et du métissage de la population (sous-entendu par l'acte sexuel) de son point de vue. Les rapports sexuels du sucrier avec les travailleuses forcées, les laboureuses des champs et en particulier les bonnes, sont eux aussi fondateurs de la population mauricienne actuelle, qui est toute entière liée, intimement, au planteur. La domination symbolique du «maître», catégorie atemporelle dans le discours de Mme R., reste vivace dans l'appropriation subjective du monde social contemporain de celle-ci. Et l'évocation dans le langage du fait sexuel comme partie prenante des rapports de production du monde de la plantation en est une manifestation concrète.

17 En relançant dans l'entretien Mme R. sur les rapports sexuels dans le monde de la plantation, celle-ci parle sans détour de la violence du maître "qui avait droit de cuissage » sur toutes les femmes, servantes et laboureuses des champs. Les épouses des maitres connaissaient ces pratiques, courantes dans la vie coloniale, mais elles n'avaient d'autre choix que de se taire, comme elle le précise. Sa dénonciation des viols passés n'a rien d'un discours nouveau de l'époque "post-coloniale ». Elle rappelle en effet les critiques moralistes des prêtres, déjà ferventes à la fin du XVIIIe siècle, qui condamnaient le viol et le "vice» des colons sur les femmes esclaves, parce qu'ils étaient aussi sources de préoccupation quant aux effets non contrôlés que les relations sexuelles entre colons et esclaves pouvaient avoir sur l'ordre colonial (Vaughan 2005).

18 Tout dans le discours de Mme R. tend plutôt à démontrer le maintien d'un ordre social colonial dans son intériorisation de la réalité sociale. La sexualité entre "maîtres » et «servantes » tient une place cruciale dans l'ordre colonial qui est subjectivé. Il faut aussi tenir compte cependant, du fait que les rapports sexuels entre le maître et la servante peuvent troubler l'intime et une famille tout entière, par-delà le seul scandale de l'existence connue d'enfants illégitimes. Ce sont des discours qui relatent un vécu personnel et familial plus distancé de "la mémoire» franco-mauricienne qui le révèlent. Aurélie par exemple, qui se définit comme appartenant aux gens de couleur avec de fortes relations dans le milieu franco-mauricien ${ }^{11}$, commence son récit personnel par l'histoire de la maîtresse de son arrière-grand-père, une bonne. Elle raconte que cette femme a finalement été rejetée par son arrière-grand-père. Or cet événement a pour Aurélie des conséquences sur sa vie personnelle : la femme devient sa nénène (nounou) et l'utilise quand elle est encore petite comme sacrifice pour pratiquer de la sorcellerie. Aurélie se constitue en sujet de sa narration et de son expérience à partir de l'histoire sexuelle de son arrière-grand-père avec la bonne. Si par conséquent les domestiques ne font jamais partie de la famille servie, y compris lorsqu'elles engendrent les enfants du maître, elles ont bel et bien une incidence sur les histoires personnelles et familiales des membres de la famille servie qui sont bousculés par les rapports sexuels de la domesticité.

19 Les propos recueillis auprès de patronnes franco-mauriciennes, ou de patronnes proches de ce milieu blanc, laissent entendre que les secrets de famille - un ancêtre fils ou fille de domestique par exemple -, sont révélés dans un contexte soi-disant plus ouvert. Aujourd'hui, «tout le monde épouse tout le monde » selon Mme R. Si tant est que les règles de l'endogamie au sein du groupe franco-mauricien soient en passe de 
s'assouplir, cela n'empêche pas les catégories raciales de se reproduire dans les imaginaires de la structure hiérarchique de la société mauricienne. Les catégories raciales actuelles pour désigner des groupes sociaux sont encore une fois liées aux représentations de la sexualité entre maîtres et servantes en tant qu'acte fondateur de la population mauricienne (ou d'une partie), du point de vue de femmes patronnes franco-mauriciennes rencontrées. Pour Mme R., la population des "gens de couleur", que d'autres assimilent dans le langage aux "mulâtres", est issue de ces rapports sexuels de la domesticité.

Mme R.: D'où, encore une fois je redis notre population dite de couleur ! Parce que toutes ces femmes qui allaient dans les maisons travailler, très souvent elles avaient des enfants avec les maîtres! Et les fils et, etc. ça c'est, énorme! Enorme! Moi-même j'ai plein d'amis qui ont des, ascendances comme ça !...C'était une manière de... ben de rester dans leur giron quoi. Elles étaient là ! Et c'était vraiment euh... fff, elles étaient là pour le maître !

Or cette interprétation historique des origines «raciales» d'un groupe de la population, né, au fond, du mélange des races blanches et noires si l'on suit le raisonnement de Mme R., repose aussi sur l'oubli, dans les mémoires du passé colonial, des moments où les catégories raciales étaient loin d'être fixées. Selon l'historienne Megan Vaughan, ce n'est pas la population, mais la catégorie raciale des "gens de couleur » qui est issue des rapports sexuels de la domesticité. La catégorie raciale a été créée au moment où ces rapports sexuels ont menacé l'ordre social, lorsque les femmes noires libres, souvent anciennes maîtresses du maître, ont commencé à gagner de l'importance numérique et du capital à la fin du XVIIIe siècle (Vaughan 2005; Allen 2011). L'héritage, des catégories raciales, n'est pas un acquis reçu du passé (Foucault 1971), mais une production du présent qui réinterprète les faits passés, ici les rapports sexuels entre maîtres et servantes. La re-fixation des appartenances sociales d'après des races supposées, mélangées ou non, est une œuvre des rapports sociaux du présent.

\section{Des rumeurs sur la sexualité des bonnes}

Il est possible que les rapports sexuels entre le Monsieur et la bonne, entre le " maitre » et la « servante ", appartiennent au passé. Mais dans les imaginaires sociaux, les bonnes sont toujours associées à l'histoire de la servante qui couche avec le patron. Et les rumeurs qui circulent à l'encontre de telle ou telle femme travaillant comme bonne deviennent parfois plus plausibles que les faits. Si la rumeur court selon laquelle untel a une relation avec son patron, c'est que l'histoire est crédible, socialement concevable (White 2000). La crédibilité de la rumeur, ou du «palabe » en kreol, s'appuie sur une convergence logique dans les représentations sociales entre le travail (dans l'intime), l'appartenance sociale racialisée et la sexualité (débridée).

Pour illustrer les rumeurs sur la sexualité des femmes qui travaillent comme bonnes, je considère le cas d'Alice, qui est une femme du milieu urbain, de la quarantaine, employée comme bonne par plusieurs couples dont une partie se classerait comme «créoles». Ils sont journalistes, cadres d'entreprise, directeurs d'établissement scolaire. Alice est entrée sur le marché du travail en tant qu'ouvrière dans une usine de textile. Elle vit actuellement avec ses trois enfants qui ont entre 10 et 18 ans et elle est séparée de son mari. On se voit pour un premier entretien chez elle, en présence de sa fille qui vaque à ses occupations dans une autre pièce fermée et non contigüe avec celle 
qu'on occupe. Alice parle pendant près de deux heures de son parcours professionnel et de son choix de quitter l'usine pour le travail de bonne, afin d'avoir plus de temps pour s'occuper de l'éducation scolaire de ses enfants. Elle évoque aussi son expérience de bonne avec ses différents employeurs. Quand je lui demande si elle a déjà eu des problèmes avec certains de ses patronnes et patrons, elle me répond par la négative. Elle m'explique qu'à chaque fois qu'elle a changé de famille, c'était parce que ses employeurs n'arrivaient plus à la payer. Quelques jours plus tard, elle me fait parvenir une lettre par le biais d'une amie dans laquelle elle m'écrit les vraies raisons pour lesquelles elle a été licenciée par trois fois par des familles "créoles». Elle me dit qu'elle a préféré l'écrire parce qu'elle cache ces évènements à ses enfants, dont l'une était présente au moment de l'entretien. Dans le premier cas, elle se fait licencier par son patron parce qu'elle «refuse de devenir sa maîtresse ». Dans les deux autres cas, bien plus détaillés, elle se fait licencier à cause d'une rumeur, qui a circulé entre patronnes, selon laquelle elle aurait une liaison avec un prêtre. Je retranscris l'un des récits écrits d'Alice sur son licenciement.

Deuxième famille : J'ai travailler pendant 3 ans ou plus, tout aller bien, je faisais que 2 jour par semaine et j'étais bien payée, sauf qu'ils étaient amis avec un prêtre et j'étais aussi amie avec et un beau jour ils ont trouvé que le [prêtre] était trop souvent chez moi. C'était vrai car il venait chez moi pour mes enfants, et il m'aidait, il me conseillais quand j'étais décourager et c'était un grand aide pour moi aide dans le sens morale et encouragement. C'est ainsi qu'ils m'ont licencié, prétextant que j'avais une liaisons avec le prêtre. Et ils m'ont pas payer. Sous prétexte qu'il a été licencié et n'a pas d'argent et qu'il me payerai après. Mais en vain.

Dans les cas relatés, il n'y a pour ainsi dire pas de rapport sexuel réel en jeu. Ce qu'Alice ne dit pas à ses enfants de son travail, et qu'elle me confie, en tant qu'étrangère à son entourage familial et social, est la rumeur selon laquelle elle aurait couché avec le prêtre. Ce sont les «palabes» qu'il s'agit de cacher, lesquels configurent son image dans les relations sociales. La circulation de l'histoire de la liaison d'Alice avec un prêtre forme sa réputation. Celle-ci détient une consistance sociale - puisqu'elle est partagée par plusieurs acteurs - qu'Alice cherche à contrôler et à limiter en n'en parlant pas à ses enfants. Je propose maintenant une interprétation des supports, idéels, imaginaires, sur lesquels la crédibilité de cette histoire repose.

Dans un autre contexte marqué par l'histoire de l'esclavage de plantation et de la colonisation, bell hooks a souligné la façon dont l'exploitation sexuelle des femmes esclaves par les maîtres des plantations avait conduit après l'abolition de l'esclavage, à la "dévalorisation perpétuelle de la féminité noire " (hooks 2015 : 103). Le viol des femmes esclaves, comme arme d'assujettissement de l'ensemble des esclaves, constitue la face matérielle du rapport social de race à partir duquel les images des femmes noires telles « la putain, la salope, la prostituée » se sont cristallisées (hooks 2015 : 104). D'autres travaux comme ceux de Jennifer Morgan montrent que l'idéologie raciste s'est développée à partir des idées préconçues des premiers voyageurs européens en Afrique sur les corps et les sexualités monstrueux des femmes africaines (Morgan 2004). Que l'esclavage soit premier aux présupposés racistes ou l'inverse, les deux s'alimentent et l'idéologie raciste résiste à l'abolition de l'esclavage. Les imaginaires de race, de genre et de sexualité se sont trouvés articulés à partir de la scène concrète des rencontres dans le travail, dans le champ de la plantation ou dans la maison des colons. Ainsi pour Karen Hansen, à propos du contexte colonial zambien, c'est particulièrement la 
position de la servante africaine qui inspire les constructions articulées des notions de genre, de race et de sexualité dont les femmes blanches sont des protagonistes principales (Hansen, 1990). Et les idéologies imbriquées de genre et de sexualité, qui sont constitutives du regard colonial sur l'autre (Sanna \& Varikas 2011), persistent après la décolonisation (Hansen 1990, Quijano 2007, Sanna \& Varikas 2011).

Si l'on revient maintenant sur le cas d'Alice, celle-ci est identifiée dans les représentations sociales comme "Créole", une catégorie ethnique qui renvoie aux descendants d'esclaves africains ${ }^{12}$ et qui repose sur l'attribution de caractères somatiques définis comme "noirs». Elle est de plus bonne. Elle vit également seule avec ses enfants. Cela est suffisant pour rendre l'histoire de sa liaison avec le prêtre crédible dans un contexte où les représentations sur le penchant des esclaves et des descendants d'esclaves pour la luxure ont demeuré (Ng Tat Chung et Romaine 2010). L'Église catholique s'est longtemps efforcée de légitimer l'entreprise de "civilisation " des esclaves émancipés et de leurs descendants durant la seconde moitié du XIXe siècle, en produisant des images négatives sur les hommes noirs libres et particulièrement sur les femmes, enclin. e. s à "l'oisiveté, l'intempérance et la luxure» (Colson 1980). Les préjugés contemporains sur les pratiques sexuelles des femmes, en particulier dans un travail marqué par l'histoire de l'esclavage, mobilisent à nouveaux frais la production idéologique raciste qui a servi à gouverner la société esclavagiste, puis postabolitionniste.

Il y a toutefois un autre aspect à prendre en compte, qui touche à la façon dont la rumeur est utile dans le contrôle des relations de service domestique par les patronnes. Alice transgresse en vérité le rapport de classe, en étant amie avec un prêtre que ses employeurs, créoles de la petite bourgeoisie urbaine, fréquentent. Or la relation de service domestique repose toujours sur un rapport hiérarchique, même si bonnes et patrons font partie du même groupe ethnique. Avoir une bonne sert toujours de preuve de son appartenance de classe, dans les conversations, les invitations à dîner, etc. L'usage des services d'une bonne aide à «se blanchir » dans les rapports sociaux. Mais si la bonne fréquente les mêmes amis que les patrons, toutes les dimensions symboliques de sa présence dans la maison sont annulées. L'invention de la rumeur sur Alice et sa sexualité avec le prêtre conduit à la «renoircir» et à recréer ainsi la différence sociale perdue entre bonnes et patronnes, en remobilisant des préjugés à la fois sexuels et raciaux. La récupération de la construction coloniale de la femme noire à la sexualité débridée par le "palabe " est une opération sociale qui n'intervient que pour rétablir une frontière de classe dans la relation de service domestique. Le « retour en arrière » dans les représentations coloniales est au bout du compte un moyen d'aller de l'avant dans la naturalisation de hiérarchies sociales qui sont en fait en train de bouger sur la scène domestique.

\section{Conclusion}

Quelles perspectives peut-on tirer de cette recherche exploratoire sur la sexualité dans les faits de langage sur les services domestiques à l'île Maurice?

Les dimensions psycho-sociales de la domination dans les services domestiques forment un pilier des systèmes sociaux hiérarchiques, comme le souligne Judith Rollins (1990). Dans ces dimensions psycho-sociales de la domination, on voit que les rapports sexuels avérés, simulés et de nature variée entre bonnes et patrons demeurent une part 
éventuelle réelle et imaginée du travail de bonne à l'île Maurice. Les actes de parole (Bornand \& Leguy, 2013) analysés sur la sexualité en relation au service domestique des implicites de discours, des mémoires du passé colonial évoquées, des rumeurs -, sont truffés de mots qui prouvent l'intériorisation de catégories raciales héritées de l'époque coloniale de la plantation. L'association des rapports de travail de service domestique à la sexualité et à des rapports de race dans le langage tend à montrer que les catégories ethniques à l'île Maurice («Créole » et « Hindoue » en particulier) ${ }^{13}$, n'ont pas remplacé ni fait disparaitre les subjectivations raciales de la hiérarchie sociale.

L'analyse des discours de bonnes et de patronnes montre aussi que les héritages coloniaux de la construction hiérarchique des différences sont plus complexes que ce à quoi on pouvait s'attendre. Le "Monsieur blanc» est parfois identifié comme le personnage qui est au sommet de la hiérarchie sociale et qui est potentiellement source de revenu contre un échange de services pour certaines bonnes. Mais tel n'est pas le cas pour les plus jeunes femmes de l'enquête qui travaillent dans les emplois domestiques. Tout dans le discours de Mme R. laisse entendre que les rapports sexuels entre « servantes » et «maitres » participent de l'ordre colonial qui a produit la population mauricienne actuelle. Mais des discours plus personnels de patronnes révèlent aussi que ces rapports ont bouleversé des familles et leur quotidien domestique. La rumeur traite Alice de "putain créole", mais Alice a depuis refait sa clientèle de services ménagers et continue d'être amie avec le prêtre, ce qui avait à un moment donné défrayé la chronique.

Pas plus aujourd'hui qu'hier, les rapports coloniaux reproduits ne sont à l'abri des troubles de l'ordre social introduits par le jeu dynamique des rapports sociaux sur la scène domestique de travail. C'est tout l'intérêt de rendre compte des points de vue différenciés de bonnes et de patronnes, mais aussi de bonnes entre elles, jeunes ou âgées. Ces points de vue différenciés montrent que les intériorisations de la réalité sociale dans le contexte de transformation actuel de la société mauricienne ne sont pas toujours en accord avec l'univers symbolique commun hérité des époques coloniales. Mais que l'on ne s'y trompe pas. C'est justement là où ces tensions et décalages de réalités sociales subjectives s'observent que les catégories raciales sont réintroduites pour légitimer la hiérarchie sociale, précisément dans les relations de service domestique ici. Dans une société qui évolue au gré des inflexions économiques liées au capitalisme global, ce n'est donc pas dans les mariages « inter-raciaux » internes à une même classe sociale qu'il faut chercher les héritages du racisme colonial, mais dans les rapports de travail et les tentatives de contrôle des travailleur.se. s pour préserver des intérêts de classe.

\section{BIBLIOGRAPHIE}

ALLEN, Richard B.. 2011. « Marie Rozette and Her World: Class, Ethnicity, Gender, and Race in the Late Eighteenth- and Early Nineteenth- Century Mauritius » Journal of Social History, 45 (2) : 345-365. 
ARONDO Maria. 1975. Moi, la bonne. Paris : Stock 2.

BORNAND Sandra \& LEGUY Cécile. 2013. Anthropologie des pratiques langagières. Paris : Armand Colin.

BOUDET Catherine \& PEGHINI Julie. 2008. « Les enjeux politiques de la mémoire du passé colonial à l'île Maurice » Transcontinentales, $6: 13-36$.

BRITES Jurema. 2000. Afeto, desigualdade e rebeldia. Bastidores do serviço doméstico. Thèse de doctorat, Universidade Federal do Rio Grande do Sul, Porto Alegre.

CHAZAN-GILLIG Suzanne. 2001. «Les fondements du pluriculturalisme mauricien et l'émergence d'une nouvelle société. Autochtonie, créolité » Journal des Anthropologues, 87 [En ligne], mis en ligne le 03 novembre 2010, consulté le 23 avril 2018. URL : http://journals.openedition.org/jda/ 2754.

CLAVEYROLAS Mathieu. 2017. Quand l'hindouisme est créole. Plantation et indianité à l'île Maurice. Paris : Editions de l'EHESS.

COCK Jacklyn. 1989. Maids and Madams. Domestic Workers under Apartheid. London : Women's Press. COLSON Didier. 1980. Approches de la pratique missionnaire catholique à l'île Maurice entre 1840 et 1895. Thèse de l'Institut Catholique de Toulouse.

COROSSACZ Valeria Ribeiro. 2014. « Cor, classe, gênero : aprendizado sexual e relações de dominio » Revista Estudos Feministas, 22 (2) : 521-542.

DAVIS Angela. 1983. Femme, race et classe. Paris : Des femmes.

DELPHY Christine. 1978. « Travail ménager ou travail domestique ? ", in Les femmes dans la société marchande, MICHEL, Andrée (dir.). Paris : Puf, 39-54.

FOUCAULT Michel. 1971. Nietzsche, la généalogie, l'histoire. Paris : Puf.

FOX-GENOVESE Elizabeth. 1988. Within the Plantation Household. Black and White Women of the Old South. Chapel Hill and London: University of North Carolina Press.

FRAISSE Geneviève. 2009. Service ou servitude : Essai sur les femmes toutes mains. Lormont : Le Bord de l'Eau.

GUILLAUMIN Colette. 1992. Sexe, race et pratiques de pouvoir. L'idée de nature. Paris : Côté-Femmes.

HANSEN Karen Tranberg. 1990. « Body Politics: Sexuality, Gender, and Domestic Service in Zambia » Journal of Women's History, $21: 120-142$.

hooks bell. 2015. Ne suis-je pas une femme? Femmes noires et féminisme. Paris : Editions Cambourakis. MARTIN-FUGIER Anne. 1979. La place des bonnes. La domesticité féminine à Paris en 1900. Paris : Editions Grasset et Fasquelle.

MORGAN Jennifer. 2004. Laboring Women. Reproduction and Gender in New World Slavery. Philadelphia : University of Pennsylvania Press.

MOUJOUD Nasima. 2007. Migrantes seules et sans droits au Maroc et en France.Dominations imbriquées et résistances individuelles. Thèse de doctorat, Paris, EHESS.

N'GAIDE Abderrahmane. 2003. «Stéréotypes et imaginaires sociaux en milieu haalpulaar. Classer, stigmatiser, toiser » Cahiers d'Etudes Africaines, $172:$ 707-738.

NEVELING Patrick. 2016. «Beyond Sites and Methods : The Field, History, Global Capitalism » in Routledge Companion to Contemporary Anthropology, S. COLEMAN, S. HYATT, A. KINGSOLVER (dir.). London : Routledge, 72-91. 
QUIJANO Anibal. 2007. «"Race” et colonialité du pouvoir » Mouvements, 51 : 111-118.

ROLLINS Judith. 1990. « Entre femmes. Les domestiques et leurs patronnes » Actes de la recherche en sciences sociales, 84 (1) : 63-77.

ROMAINE Alain \& NG TAT CHUNG Serge. 2010. Les créoles des idées reçues : origines du racisme antiafricain à l'T̂le Maurice. Beau Bassin : Editions Marye-Pike.

ROMERO Mary. 1992. Maid in the USA. New York : Routledge.

RUBENSTEIN Steven. 2007. "Circulation, accumulation and the power of Shuar shrunken heads » Cultural Anthropology, 22 (3) : 357-399.

SANNA Maria Eleonora \& VARIKAS Eleni. 2011. «Genre, modernité et "colonialité du pouvoir” : penser ensemble des subalternités dissonantes » Cahiers du Genre, 50 : 5-15.

SIMMEL Georg. 1999. «Excursus sur la frontière sociale », in Sociologie. Etudes sur les formes de la socialisation. Paris : Presses Universitaires de France, 609-612.

STOLER Ann Laura. 2002. Carnal Knowledge and Imperial Power: Race and the Intimate in Colonial Rul., Berkeley : University of California Press.

TABET Paula. 2004. La grande arnaque. Sexualité des femmes et échange économico-sexuel. Paris :

l'Harmattan.

TEELOCK Vijaya. 1998. Bitter Sugar: Sugar and Slavery in 19th Century Mauritius. Mauritius : Mahatma Gandhi Institute.

VAUGHAN Megan. 2005. Creating the Creole Island. Slavery in Eighteenth-Century Mauritius. Durham and London : Duke University Press.

WHITE Luise. 2000. « Telling more : lies, secrets and history » History and Theory, 39:11-22.

\section{NOTES}

1. Cette recherche s'inscrit dans le projet de recherche ERC « The Colour of Labour. The racialised lives of migrants » coordonné par Cristiana Bastos à l'Institut des Sciences Sociales de Lisbonne, lequel interroge les processus de racialisation au travail.

2. Ce sont les termes en usage à l'île Maurice actuellement. Tous les mots entre guillemets sont des données émiques tirées des discours des personnes rencontrées. Les données émiques sont centrales à l'analyse que je propose des faits de langage sur la sexualité comme faits sociaux.

3. Elles racontent notamment des anecdotes mettant en scène ce qu'elles ont refusé de faire à la demande de leur patronne ou patron.

4. J'enquête dans deux types d'espace où le marché des services domestiques est particulièrement dynamique à l'île Maurice : les zones urbaines et les zones côtières. L'enquête en milieu urbain est concentrée sur Curepipe, et l'enquête en milieu côtier est menée sur la côte ouest qui est sujette à une intense urbanisation depuis les années 2000.

5. «Je travaille dans la maison de Madame."

6. La dépendance désigne le logement de la servante et de sa famille dans la cour de la maison servie.

7. Type de logement à bas prix construit par l'Etat.

8. Les rémunérations sont réévaluées tous les ans.

9. Tous les mots entre guillemets sont les leurs.

10. Les passages masqués visent à préserver l'anonymat.

11. Elle est âgée de la cinquantaine et fait partie d'une famille au fort capital économique. 
12. Ce qui n'est d'aucune précision historique, il s'agit bien d'une catégorisation de l'autre. Voir les travaux de Vijaya Teelock en particulier sur ce point (1998).

13. Lesquelles ont été créées, il faut le rappeler, par l'administration coloniale britannique pour empêcher les alliances politiques sur la base de la classe (Neveling 2016).

\section{RÉSUMÉS}

Cet article ne traite pas tant des rapports sexuels comme dimension du travail de service domestique, que de la sexualité comme objet de discours sur les relations de service domestique dans la société mauricienne contemporaine. L'analyse porte précisément sur la façon dont le fait de langage sur l'aspect sexuel des relations de service domestique ordonne le social et légitime les hiérarchies actuelles, en puisant dans les catégories raciales héritées de l'époque coloniale de la plantation sucrière. La démarche, exploratoire, confronte les discours de "bonnes » et de "patronnes» qui évoquent de manière implicite, par leurs mémoires du passé colonial ou les rumeurs dont elles font l'objet, les imaginaires de race et de sexualité imbriqués au travail de bonne. Les propos analysés donnent à voir la complexité des héritages coloniaux de la construction hiérarchique des différences qui émerge de la réintroduction des catégories raciales là où les rapports sociaux se transforment.

Instead of focusing on sexual relations in domestic service work, this article is about sexuality as an object of discourse on domestic service relations in Mauritian contemporary society. Is questioned the way the fact of speaking on the sexual aspect of domestic service relations does order and legitimate the current social hierarchies, by turning back to the racial categories of colonial inheritance. With an exploratory approach, the analysis confronts the discourses of some "maids" and "Madams" which bring together the social imaginaries of race and sexuality around the topic of maid's work. The empirical data analysis helps to grapple with the complexity of colonial heritage that emerges from the reintroduction of racial categories exactly where the social relations are refurbished.

\section{INDEX}

Mots-clés : service domestique, catégories raciales, héritage colonial, imaginaires sociaux, sexualité

Keywords : domestic service, racial categories, colonial heritage, social imaginaries, sexuality

\section{AUTEUR}

\section{COLETTE LE PETITCORPS}

Postdoctorante, ERC AdG 2015 - 695573 The Colour of Labour, PI Cristiana Bastos, Institute of Social Sciences, University of Lisbon, Av Anibal Bettencourt, 9, 1600-189 Lisbon, Portugal. Colette Le Petitcorps est docteure en sociologie. Elle est actuellement post-doctorante à l'Institut des Sciences Sociales de Lisbonne dans un programme de recherche ERC Advanced Grant intitulé 
«The Colour of Labour. The racialized lives of migrants » dirigé par Cristiana Bastos. Ses recherches portent sur les services domestiques, les migrations et l'articulation des rapports sociaux en France et à l'Ile Maurice. 